BIJDRAGEN TOT DE KENNIS DER FAUNA VAN CURAÇAO.

Resultaten eener Reis van Dr. C. J. VAN DER HORST in 1920.

\title{
UEBER EINIGE VON Dr. C. J. VAN DER HORST BEI CURAÇAO GESAMMELTE MEDUSEN
}

\author{
VON
}

\section{Dr. GUSTAV STIASNY.}

(Mit 7 Textfiguren.)

Die kleine Scyphomedusen-Sammlung, die Dr. vAN DER Horst auf Curaçao erbeutet hat, umfasst 28 Exemplare, die 3 verschiedenen Genera und Species angehören u. zw.

Aurelia aurita (Linneus) Lamarck.

Pelagia curaçaoensis spec. nov.

Cassiopeia van der. Horsti spec. nov.

Das Material ist interessant, zunächst weil die pelagische Fauna von Holländisch Westindien überhaupt nur wenig erforscht ist, dann aber auch wegen der Fundorte. Die Exemplare von Aurelia stammen aus Spanisches Wasser, einer kleinen Bucht auf Curaçao, die nur durch einen schmalen Canal (Spanischer Hafen) mit der offenen caraibischen See in Verbindung steht. Aus Spanischer Hafen stammen die Pelagia, die Cassiopeia-Exemplare schliesslich aus einem kleinen Mangrove-Tümpel im innersten Teile der Caracas-Bai. Ueber die eigenartigen biologischen Verhältnisse der Fundstellen vergl. das Narrative (15, p. 7 ).

Genus Aurelia Péron Lesueur.

Aurelia aurita (Linneus) Lamarck.

Spanisches Wasser: 11. V. 20, 3 Exemplare von 230, 250, $280 \mathrm{~mm}$. Schirmbreite $\mathrm{N}^{0} 205^{1}$ ).

Färbung: bläulich-rosa, Gonaden (geschlechtsreif) und Schirmrand lichtviolett. Die Kanäle verlaufen gestreckt, regelmässig, mit wenigen peripheren Anastomosen. Zumeist 3 Kanalwurzeln per Oktant

Genus Pelagia Péron Lesueur.

Pelagia curaçaoensis spec. nov.

Spanischer Hafen: 6. V. 20, 3 Exemplare $\mathrm{N}^{0}$ 224. (Textfig. 1).

Diagnose: Schirm doppelt so breit als hoch, am Scheitel abgeflacht, an den Seiten steil abfallend oder etwas convex, Gallerte dick. Zahlreiche verhältnismässig grosse Nesselwarzen auf der ganzen Exumbrella, in unregelmässigen Reihen angeordnet: am Apex rundlich, hoch, an den Seiten elliptisch oder gestreckt, oft sehr lang $(2-3 \mathrm{~mm})$, am Schirmrand flach, sämmtlich mit sehr dichten Querfalten, mit oder ohne Längskamm. Randläppchen quadratisch, schwach eingebuchtet; Mundarme kurz, nicht oder nur wenig über den Schirm hervorragend, mit schmalem, schwach gekräuseltem Hautsaum. Auf den Pfeilern des Mundrohrs und auf der Mittelrippe der Arme kleine rundliche Häufchen von Nesselzellen.

1) Inventar. $N^{0}$ des Rijks-Museums. 
Grösse: $30-32 \mathrm{~mm}$ Schirmbreite, $13-15 \mathrm{~mm}$ Schirmhöhe.

Magenröhre $=1 / 4-1 / 2$ Schirmradius, Mundarme $\pm r$, Tentakel $\pm r$.

Farbe: Gallerte durchsichtig, gut entwickelte Gonaden tiefrosa, Magen, Radialtaschen und Mundarme lichtrosa, Nesselwarzen auf der Exumbrella gänzlich ungefärbt, Nesselzellhäufchen auf den Pfeilern des Mundrohrs und Armen weisslich.

Nach BIGELow ist „for the present the chief duty of any one, who can examine a small series of Pelagia (is) to give details of the two characters, which according to MAAS (1903) and MAYER (1910) separate the "species", i. e. proportion of manubrium and moutharms, and form of the exumbral nettle knobs" (2, p. 88).

Die Untersuchung der vorliegenden 3 Exemplare zeigt aufs neue, wie unsicher und variabel diese Merkmale sind. Denn nicht nur, dass das Verhältnis der Länge des Manubriums und der Mundarme je nach dem Kontractionszustand wechselt $(13$, p. 36,2, p. 89), ist, besonders bei so kleinen Exemplaren, ein genaues Messen kaum möglich. Jeder Untersucher wird wohl andere Zahlenwerte angeben.

Dass aber auch das Hauptmerkmal, die Form und Beschaffenheit der exumbralen Nesselwarzen unzuverlässig ist, geht daraus hervor, dass diese sich nicht nur mit zunehmendem Alter verändern,

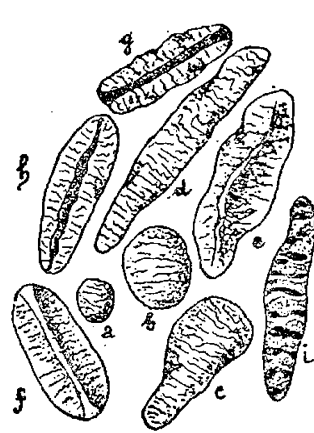

Fig. 1. - Pelagia curacaoensis nov. spec.
Nesselwarzen auf der Exembrella.
Exem sondern auch bei ein und demselben Individuum je nach der Lage auf der Exumbrella ganz verschieden sind (2, p. 89). Die 3 Exemplare von Curaçao zeigen zwar zumeist recht deutlich feine Querfaltung, wie solche von VANHöFFEN nur bei Pelagia minuta angegeben wird. Während die Nesselwarzen jedoch bei dieser species klein, rundlich, ohne Längskamm sind, kann man bei Pelagia curaçaoensis rundliche hohe (Textfig. 1, $a, b$ ) am Apex, längliche wurstförmige ohne Längskamm $(c, d)$ und mit Längskamm $(e, f)$, mit Längsfurche $(g, h)$, endlich solche mit nur wenigen breiten Querwülsten $(i)$ an den Seiten beobachten. Also an ein und demselben Exemplar ganz verschiedene (vielleicht noch mehr als beschrieben) Formen der Nesselwarzen, je nach ihrer Lage. Gegen den Schirmrand werden sie ein wenig flacher. - Die Gonaden sind bei den 3 Exemplaren, die ziemlich gleichaltrig sind, gut entwickelt, jedoch nicht geschlechtsreif. Möglicherweise liegen daher Jugendstadien vor.

Aus den westindischen Gewässern selbst ist bisher keine Pelagia-Species bekannt, doch sind zahlreiche Arten einerseits in den Gewässern von Florida bis Cap Cod, andrerseits bei Pernambuco und in der Tropenzone des atlantischen Oceans nachgewiesen worden.

Am nächsten verwandt scheint die neue Form von Curaçao mit 2 kleinen Pelagia-Species zu sein, die aus dem letzteren Verbreitungsgebiet stammen, was ja ohne weiteres verständlich ist, da die Südaequatorial-Strömung resp. die Antillen-Strömung eine Verbindung herstellt.

Pelagia minuta Vanhöffen, von Pernambuco, ist kleiner, flacher, hat ein verhältnismässig langes Mundrohr, längere Mundarme. Die Nesselwarzen sind wie bei $P$. curaçaoensis mit dichter Querfaltung. versehen, jedoch ohne Längskamm. (In der Beschreibung 12, p. 12-22, spricht VANHöFFEN nur von kleinen rundlichen Nesselwarzen, während diese nach seinen Fig. 16, 17, Taf. VI, doch auch länglich sind.)

Pelagia crassa Vanhöffen hat wohl eine sehr ähnliche Körperform (12, Taf. I, Fig. 1, 2), dicke Gallerte, übereinstimmende Form der Randläppchen, jedoch viel längere Mundarme und ganz anders beschaffene Nesselwarzen: flach, mit Längskamm und wenigen undeutlichen Querfalten (12, Taf. VI, Fig. 13-15). Fundort: atlantischer Ocean, zwischen Afrika und Südamerika.

Pelagia minuta Vh. wird von MAYER $(8$, p. 576) als eine Varietät oder als ein Jugendstadium von P. phosphora Haeckel angesehen, indem er die dichte Querfaltung der Nesselwarzen als "due to contraction?", als eine Folge der Alkoholconservierung betrachtet. Ich glaube kaum, dass bei den gutconservierten (Formaldehyd) Exemplaren von P. curaçaoensis die Querfaltung der Nesselwarzen auf eine Schrumpfung zurückzuführen ist. Dass es sich hier jedoch möglicherweise um Jugendstadien eines anderen Species, am ehesten noch von $P$. phosphora Haeckel, wohl kaum von $P$. cyanella handelt, 
die ja gleichfalls in der Tropenzone des atlantischen Oceans nachgewiesen sind, wäre ja immerhin denkbar.

Kleine Exemplare von Pelagia, die nicht geschlechtsreif sind, mit einiger Sicherheit zu bestimmen, ist gegenwärtig kaum möglich.

\section{Genus Cassiopeia Péron Lesueur. \\ Cassiopeia van der Horsti nov. spec.}

(Textfig. 2, 3, 4, 7.)

Caracas-Bai: in einem Mangrovetümpel, 22 Exemplare: № 204.

Diagn ose: Schirm flachgewölbt, mit niedriger centraler Kuppel, 14-18 (meist 16) Rhopalien, Exumbrella ohne farbige Zeichnung mit feinen radiären Streifen und Furchen und breitem peripherem Ringwulst, Subumbrella ohne Flecken, 3-5 (meist 3) Velarläppchen pro Sektor, Ocellus ohne Pigment, Mundarme $\pm 1 \frac{1}{2} \mathrm{r}$ lang, fiederig-dichotom mit zahlreichen kleinen und wenigen grossen Kolbenblasen, sinusartige Anschwellung der meist 32 Rhopalarcanäle im äusseren Drittel des Verlaufes. ArmscheibenDurchmesser: $\pm 2 / 3$ r. Grösse: Schirmbreite maximal (?) $170 \mathrm{~mm}$. Färbung: gelblich-bräunlich, durchscheinend, mit braunen grossen Kolbenblasen auf den Mundarmen.

Hauptmerkmale: An Stelle der Farbenzeichnung (weisser Ring auf der Exumbrella, weisse Flecken auf Ex- und Subumbrella), eigenartige Struktur (radiäre feine Streifen und Furchen, Ringwulst), lange Mundarme $\left(+1 \frac{1}{2} \mathrm{r}\right)$, sinusartige Anschwellung der Rhopalarcanäle, \pm 16 Rhopalien.

Aus den westindischen Gewässern sind bisher zwei Cassiopeia-species bekannt, $C$. xamachana Bigelow und $C$. frondosa (Lamarck). Von beiden species unterscheidet sich unsere Form durch eine ganze Reihe von Merkmalen, die aus folgender Tabelle ersichtlich sind; die auffallendsten Unterschiede sind wohl der Mangel der Farbenzeichnung auf der Exumbrella, ihre Struktur und die langen Mundarme.

\begin{tabular}{l|l|l|l}
\multicolumn{5}{l}{ Cassiopeia } \\
\hline \hline & \multicolumn{1}{|c|}{ xamachana } & \multicolumn{1}{c}{ frondosa } & \multicolumn{1}{c}{ van der Horsti } \\
\hline Grösse & bis $240 \mathrm{~mm}$ & bis $260 \mathrm{~mm}$ & bis $170 \mathrm{~mm}$ \\
\hline Form der Exumbrella & concav & flach & flach mit centraler Kuppel \\
\hline Struktur & glatt & glatt & $\begin{array}{l}\text { mit Radiär-Struktur und Ring- } \\
\text { wulst }\end{array}$ \\
\hline Zahl der Rhopalien & $11-23( \pm 16)$ & 12 & $14-18( \pm 16)$ \\
\hline Ocellus & rotbraun & fehlt & fehlt \\
\hline Länge der Mundarme & $\left.\mathrm{r}-1.25 \mathrm{r}^{1}\right)$ & $\left.3 / 4 \mathrm{r}-\mathrm{r}^{1}\right)$ & $\mathrm{r}- \pm 11^{1 / 2} \mathrm{r}$ \\
\hline Kolbenblasen & kleine und grosse & nur kleine & kleine und grosse \\
\hline Magen & "cruciform" & quadratisch $\left.{ }^{2}\right)$ & kreisrund \\
\hline Radiärcanäle & gleichmässig dick & gleichmässig dick & $\begin{array}{l}\text { mit sinusartiger Anschwel- } \\
\text { lung im äusseren Drittel }\end{array}$ \\
\hline Färbung des Schirmes & grünlich-graublaumitweissen \\
& Flecken und weissem Ring & $\begin{array}{l}\text { elfenbeinfarben bis grünlich } \\
\text { mit weissen Flecken und } \\
\text { weissem Ring }\end{array}$ & $\begin{array}{l}\text { gelblich bräunlich ohne } \\
\text { Zeichnung }\end{array}$ \\
\hline
\end{tabular}

Im ganzen steht die neue Form also der C. xamachana Bigelows näher als der C. frondosa Lam. Vielleicht ist sie identisch mit jener von MAYER (8, p. 643) als Farbenvarietät von xamachana beschriebenen seltenen Form, ,in which the spoke - like, dull white spots are diamond-shaped, and there is no broad, white ring on the exumbrella. The whole medusa is more translucent than are the more abundant medusae with the white ring. They are also smaller than the common form. Curiously enough this color variety bears a striking resemblance to Cassiopeia ndrosia Ag. and Mayer, from the Fiji Islands, South Pacific”.

1) Ich folge hier den Angaben MAYERS (8); diejenigen von MAAS (7; p. 40) stimmen damit nicht überein. 2) Vergl. die Abb. 1, Pl. XIII, Fig. 2, 4, 5 . 
Der Mangel der Zeichnung auf der Exumbrella ist nichts ungewöhnliches unter den Cassiopeiaspecies. Wir finden denselben bei einer ganzen Reihe ostafrikanischer und malayischer Cassiopeia-Arten wieder, so z.B. bei Cassiopeia andromeda Hartlaubs von Djibuti, C. depressa Haeckel von Madagascar, C. andromeda var. maldivensis Browne von den Malediven und $C$. andromeda var. malayensis Maas aus dem malayischen Archipel. Uebrigens zeigt die schöne Abbildung von AGassiz (1, Taf. XIII, Fig. 1) von $C$. frondosa gleichfalls keine Farbflecke.

Auffallend ist jedoch, dass der für $\mathcal{C}$. van der Horsti so charakteristische periphere Ringwulst auf der Exumbrella auch von HaRTlaub bei $C$. andromeda von Djibuti, von BRowne bei $C$. $a$. var. maldivensis nachgewiesen wurde. Ich fand denselben übrigens auch bei den in meinem Besitz befindlichen Originalen von $C$. andromeda var. malayensis Maas wieder - von MAAs in seiner Beschreibung (7) nicht erwähnt. - Wahrscheinlich ist auch bei $C$. frondosa diese eigentümliche Struktur vorhanden wie aus MAYERS Abbildung (8, Taf. 72, mittleres unteres Exemplar) hervorgeht, wurde jedech weder von Agassiz noch von MAYER erwähnt. Der Ringwulst scheint überall da vorhanden zu sein, wo der weisse Ring auf der Exumbrella fehlt. Die feinen Radiärstreifen und Furchen auf derselben werden nirgends erwähnt. Aehnliche Radiärstructur wurde von Haeckel $(6, p .577)$ bei den Polyrhiza-species beschrieben. Auch die Mundarme stellen - namentlich gegenüber den beiden amerikanischen Formen ein gutes Erkennungszeichen dar, da sowohl xamachana als frondosa viel kürzere Mundarme besitzen. Mundarme von $\pm 1 \frac{1}{2}$ r Länge sind nur bei wenigen Cassiopeia-species aus ganz anderen Verbreitungsgebieten bekannt, so bei $C$. ornata var. digitata Maas aus dem malayischen Archipel und $C$. Mertensii Brandt und ndrosia Ag. \& May. aus dem Pacifik.

Schliesslich sind bei keiner anderen Form sinusähnliche Anschwellungen der Rhopalarcanäle beschrieben:

Am besten scheint $C$. van der Horsti von Curaçao überein zu stimmen mit der durch HaRTLAUB beschriebenen $C$. andromeda von Djibuti (Ostafrika) (5, p. 467). Sie unterscheidet sich von derselben

1) durch ihre bedeutendere Grösse $(150-170 \mathrm{~mm}$ gegenüber $90-100 \mathrm{~mm})$,

2) durch die grössere Länge der Mundarme $\left( \pm 1 \frac{1}{2}\right.$ r gegen $\pm \mathrm{r}$ ),

3) durch die sinusähnlichen Anschwellungen im äusseren Drittel des Verlaufes der Rhopalarcanäle.

Von den durch Dr. vaN DER HORST gesammelten 22 Exemplaren messen die grössten 150-170 mm Schirmbreite, die jüngsten Stadien 35 und $40 \mathrm{~mm}$ Schirmdurchmesser. Sämmtliche lassen die Zeichnung auf der Exumbrella vermissen. Sie sind gleichmässig gelblich-bräunlich mit bräunlichen grossen Kolbenblasen. Die Zeichnung ist nicht etwa verblasst; dann müssten doch Spuren davon sichtbar sein, sondern die Objekte zeigen laut Aussage des Finders ihre natürliche Färbung.

'Bei der eigenartigen Struktur der Exumbrella handelt es sich um eine „ungefähr auf der Grenze zwischen dem 3. und äussersten Viertel des Scheibenradius befindliche schmale niedrige sich haupt-

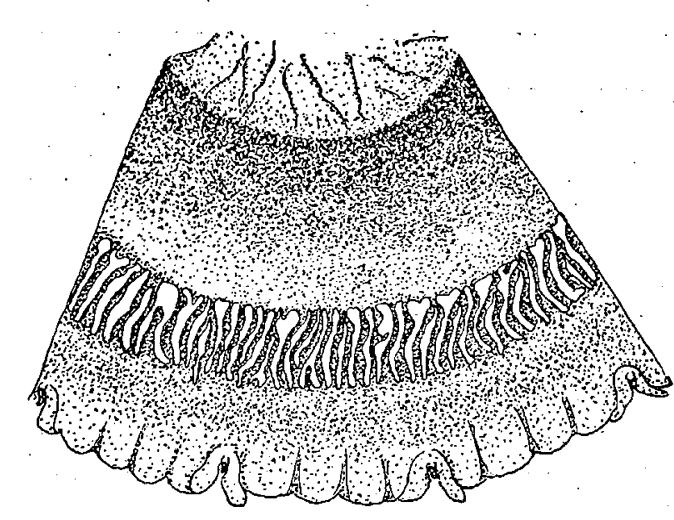

Fig. 2. - Cassiopeia van der Horsti nov. spec. Teil der Exumbrella eines jugendlichen Exemplars von circa $30 \mathrm{~mm}$ Schirmbreite. sächlich durch histologische Differenzierung abhebende ringförmige Erhebung. Auf diesem Ringwulst zeigen sich viele kurze unregelmässige Radialfalten der dorsalen Gallerte. Seiner Lage nach entspricht er dem milchweissen Ring, der von C. polypoides Keller und C. xamachana Bigelow beschrieben wurde und der äusseren Umrandung der grossen saugnapfähnlichen zentralen Vertiefung auf dem Scheibenrücken erwachsener Exemplare"' (5, p. 471).

Dieser Ringwulst ist bei jüngeren Exemplaren (Textfig. 2) deutlicher erkennbar, verhältnismässig breiter und wird durch eine grosse Anzahl radiär verlaufender Längswülste und dazwischenliegenden Rinnen gebildet. Bei älteren Individuen (Textfig. 3) ist er relativ weniger breit, die Wülste und Rinnen sind weniger deutlich erkennbar, oft ganz undeutlich, dagegen ist er durch ziemlich tiefe, scharf ausgesprochene Ringfurchen zu beiden Seiten, aussen und innen, von den benachbarten Partien der Exumbrella abgesetzt. Die Ausbildung dieser histologischen Differenzierung steht wohl unzweifelhaft in Zusammenhang mit der Function der Exumbrella als Saugnapf (4, 
p. 962). - Der mittlere Teil der Exumbrella, in der Ausdehnung etwa des Magenbodens, ist flachkuppelförmig erhöht, bildet eine runde Scheibe, deren Centrum öfters vertieft ist und die gesgenüber den angrenzenden tieferliegenden Partien der Exumbrella deutlich sich abhebt. Bei jüngeren Exemplaren finden sich hier ganz unregelmässig angeordnete kurze oder längere radiäre Furchen, während bei älteren Individuen zahlreiche in : die Gallerte eingesenkte längere oder kürzere gewundene Canälchen zu sehen sind. Ihre hellere Färbung, mehr weisslichgelblich, glaube ich auf die Anwesenheit symbiontischer Algen zurückführen zu sollen, die sonst hier nur an vereinzelten Stellen, zwischen den Saugkrausen der Mundarme, nachzuweisen sind (Textfig. 2 und 3). - Rings um den centralen flachen Dom ist die Exumbrella eingesenkt und steigt in breiter Zone gegen die Peripherie nach dem obenerwähnten Ringwulst, der am höchsten liegt, an. Aeltere Exemplare zeigen auf dieser breiten ringförmigen Zone eine Unzahl radiärverlaufender feiner Längswülste und Rinnen. Bei jüngeren ist dieser Teil der Exumbrella glatt, ebenso der Schirmrand, während letzterer bei erwachsenen Objekten unregelmässig gekörnelt oder quergefurcht und dünner als der centrale Teil ist.

Die Zahl der Rhopalien schwankt zwischen circa 14

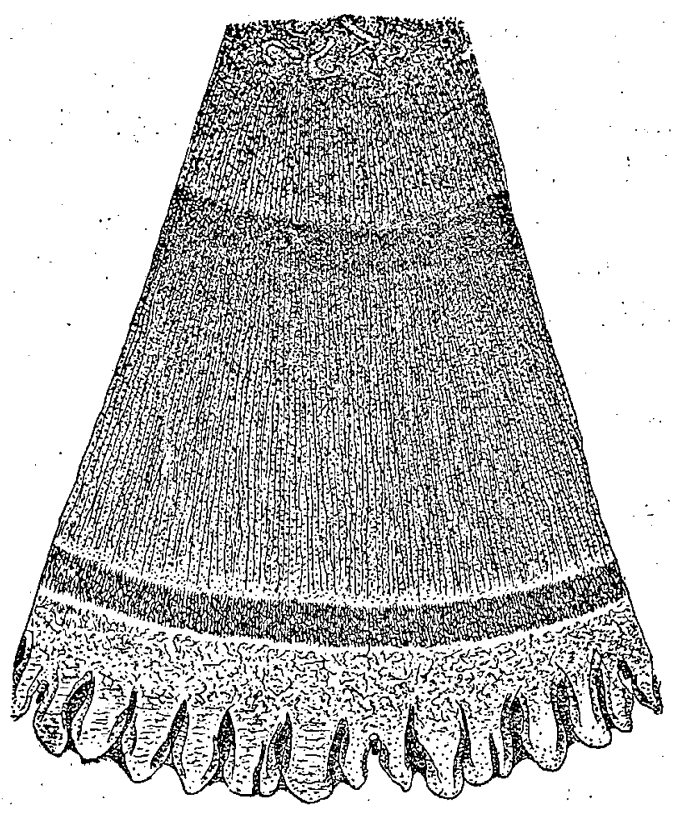

Fig. 3. - Cassiopeia van der Horsti nov. spec. Teil der Exumbrella eines erwachsenen Exemplars von circa $130 \mathrm{~mm}$ Schirmbreite (etwas verkleinert)

bei jungen, bis circa 18, meist jedoch 16, bei erwachsenen Exemplaren, ist jedoch schwer mit Sicherheit feststellbar, weil die Einkerbungen am Schirmrande stellenweise sehr schwach sind und oft fast ganzrandige Partien vorkommen. Nicht minder schwierig ist daher auch die Zahl der Velarläppchen festzustellen, von denen 3, 4, 5 zwischen je 2 Randkörpern zu zählen sind. Die jüngsten Stadien weisen 3 Randläppchen pro Sektor auf (Textfig. 2), doch zeigen auch die grössten Exemplare in manchen Parameren nicht mehr als 3. Die Rhopalarläppchen sind zumeist etwas kürzer und schmäler als die Velarläppchen. Auf die Schwierigkeit der Feststellung genauer Zahlenverhältnisse haben fast alle Autoren aufmerksam gemacht. Am ehesten sind sie entweder mit Hilfe der, allerdings auch unregelmässigen, zwischen 2 benachbarten Gallertfurchen liegenden Gallertwülste (Textfig. 3, worin Furchen und Wülste etwas übertrieben stark dargestellt sind) oder durch die Zahl der Taschen des Gefässystems festzustellen, indem jedem Velarläppchen etwa eine Vorwölbung der Taschen entspricht (7, p. 41); ähnlich, wie die Zahl der Randkörper leichter durch Feststellung der Anzahl der Rhopalarcanäle nach erfolgter Injection bestimmt werden kann, als bei dem nicht injicierten. Tier.

Die Magencontour ist stets rundlich. Ringcanal nirgends nachweisbar. Die Anzahl der Radialcanäle, meist 32, schwankt zwischen 28 und 36, je nach dem Alter: Bèi den meisten Exemplaren sieht man nach Injection von Delaf. Haematoxylin (Textfig. 4) im Verlaufe der Rhopalarcanäle, in der äusseren peripheren Hälfte eine unregelmässige langgestreckte sinusartige Verbreiterung, Fig. 4. - Cassiopeia van der Horsti 1ov. spec. die gegen die Rhopalien zu schmäler wird. Die Interrhopalarcanäle bleiben dagegen gleichmässig dick, sind etwas schmäler als die rho-

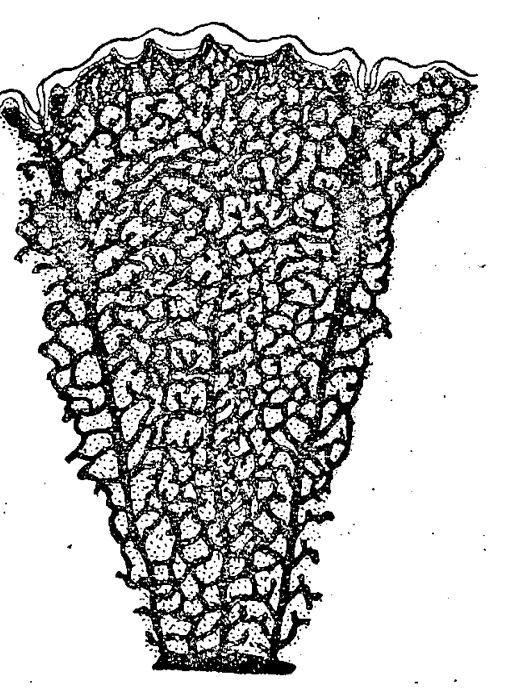
Gefässystem des Schirmes nach einem Injectionspräparat. palaren und verlieren sich etwa in der Höhe der sinusartigen Verbreiterung der Rhopalarcanäle im engmaschigen feinen Anastomosennetz (Textfig. 4).

Die Ringmuskulatur ist besonders dort, wo sie sich an die mehr centralgelegenen Fiederarkaden anschliesst, kräftig; die einzelnen Muskalblätter sehr breit.

Die $M u n d a r m e$ sind bei erwachsenen Tieren in ihren proximalen Teilen ausgesprochen fiederig, mit 6-8 und mehr Seitenästchen, distal oft sehr deutlich dichotom, was bei Injection der Gefässe noch 
mehr hervortritt. Bei jungen Exemplaren von 30-40 mm Schirmdurchmesser sind die Mundarme noch deutlicher dichotom. Die Saugkrausen stehen hier sehr dicht und bedecken die proximalen Teile völlig, während sie bei älteren Individuen viel schwächer entwickelt sind, viel weniger dicht stehen und hauptsächlich auf die Mundarmrinnen beschränkt sind. Meist sind die Mundarme 3- oder 4-ästig in ihren distalen Teilen. Ihre unregelmässige. Form entsteht durch ungleichmässiges Wachstum der verschiedenen Teile.

So habe ich z.B. einen Mundarm von $80 \mathrm{~mm}$ Länge gefunden, der so tief gegabelt ist, dass die Gabelung fast unmittelbar an der Ursprungstelle, in unmittelbarer Nähe der Armscheibe liegt. Von den beiden Gabelästen bleibt der eine einfach und trägt nur in seinem proximalen Teile einige kräftige Seitenästchen, während der andere, proximal deutlich gefiedert, sich in seinem halben Verlaufe nochmals tief gabelt.

Die Länge der Mundarme variirt von $\pm \mathrm{r}$ bei den kleinen Exemplaren bis zu $1 \frac{1}{1} 4^{-1} 1 \frac{1}{2} \mathrm{r}(\mathrm{j}$ a vereinzelt werden dieselben noch etwas länger) bei den erwachsenen, wo sie den Eindruck machen, viel schlanker, schmäler, weniger stark dorsoventral abgeplattet zu sein. Doch mag dies auch an der viel schwächeren Ausbildung der Saugkrausen bei den erwachsenen Exemplaren liegen. An jüngeren Exemplaren tritt die paarweise Anordnung der Mundarme und gleichmässige Ausbildung der Dichotomie deutlich zu Tage.

Bei den meisten Exemplaren finden sich im Centrum der Armscheibe ein oder mehrere grosse Kolbenblasen von bis $30 \mathrm{~mm}$ Länge. Sie sind oft bräunlich gefärbt, geschlossen, ohne äussere Oeffnung, oder breite Säcke mit weitem Lumen und klaffender Oeffnung, oder wieder spitzzulaufende Anhänge ohne Oeffnung, endlich breite handförmige Blätter. Auf den Mundarmen sind sie zumeist viel kleiner, bis $20 \mathrm{~mm}$ lang, geringer an Zahl und regelmässig dort $\mathrm{zu}$ finden, wo eine Gabelung oder Verzweigung der Mundarme eintritt. ZWischen den Saugkrausen überall zerstreut kleinere oder grössere, weissliche, selten bräunliche Bläschen, Stäbchen, Säckchen, die auch zu Bändern werden, kurz ein überraschender Reichtum an verschiedenen Formen der Anhänge. Dazu kommen dann noch bei weiblichen Exemplaren jene viel kleineren zarteren Bildungen, welche die Zottenrosette zusammensetzen. Bei den jüngsten Exemplaren finden sich geschlossene grosse weissliche Kolbenblasen im Centrum Ger Armscheibe und den benachbarten Teilen der Mundarme, an den distalen Teilen der letzteren nur viel kleinere längliche Bläschen, also eine viel geringere Mannigfaltigkeit an Formen wie bei den adulten. Aehnliche grosse blattförmige solide Anhänge mit compliciertem Gefässystem, wie von mir (10) bei Cassiopeia andromeda und var. malayensis beschrieben, sind hier nirgends $\mathrm{zu}$ sehen. - Der grösseren Mannigfaltigkeit der Form der Mundarmanhänge entspricht zweifellos eine Vielseitigkeit der Funktion: Fangapparat, Waffe, Atmung (Oberflächenvergrösserung), Substrat (Anheftung der jungen Brut), Schutz der Embryonen, u.s.w.

Zur Zeit liegen mir die Typen-Exemplare von 6 verschiedenen species oder Varietäten von Cassiopeia vor: ornata Haeckel von den Palau Inseln, ornata var. digitata Maas aus dem malayischen Archipel, C. andromeda var. zanzibarica Chun von Ostafrika, C. andromeda var. malayensis Maas und var. cyclobalia Schultze aus dem malayischen Archipel, und C. picta Vanh. aus dem Roten Meer, ferner zahlreiche Exemplare von $C$. andromeda von verschiedener Provenienz (Tur, Djeddah, Seychellen). Die Mehrzahl dieser verschiedenen Formen wurde von MAAS, MAYER, BROWNE und Verf. als zweifellos identisch mit $\mathcal{C}$. andromeda erklärt. Vergleicht man nun die Originale mit einander, so ist man im ersten Augenblick überrascht wie sehr die genannten Species doch von einander verschieden aussehen, indem gewisse Merkmale, wie Zeichnung der Exumbrella und Subumbrella, Färbung, grösserer oder geringerer Grad von Durchsichtigkeit, Form des Schirmes, Länge der Mundarme anscheinend dem Habitus ein charakteristisches Gepräge verleihen. Vor allem ist es die Zeichnung der Exumbrella mit ihrem weissen Ring und weissen Flecken, die bei $C$. cyclobalia und picta auffallen, die weissen Streifen auf der Subumbrella, weisse Streifen und Flecken auf den Mundarmen bei ornata, Elfenbeinfarbe oder grünliche Färbung bei andromeda, Durchsichtigkeit und Farblosigkeit bei var. malayensis (und van der Horsti). Vergleicht man jedoch die verschiedenen Exemplare ein und derselben Species, so zeigt sich schon bei einer geringen Anzahl von Individuen 
die überaus grosse Variationsbreite und damit auch Unzuverlässigkeit gerade dieses so stark ins Auge fallenden (Farben-) Merkmales. Dazu kommt noch, dass Jugendstadien verschiedener Formen, wo die Farbenunterschiede noch nicht so stark hervortreten, so sehr übereinstimmen, dass sie kaum mehr von einander zu unterscheiden sind. Das Studium der Typenexemplare wohl auch der übrigen sogenannten guten Arten von Cassiopeia dürfte also kaum zu einem einigermassen zufriedenstellenden Ergebnis führen.

Bei der geographischen Verbreitung fällt auf, dass Cassiopeia im östlichen Teil des Atlantic und im Mittelmeer nicht nachgewiesen ist (der vereinzelte Fund von $C$. $a$. bei Cypern durch MAAS (7, p. 46) ist wohl nur als ein Ausnahmsfall zu betrachten). Es lassen sich daher zwei räumlich scharf getrennte Verbreitungsgebiete von $C$. feststellen: 1) als $\mathrm{Hauptverbreitungsgebiet} \mathrm{das}$ indopacifische mit $C$. andromeda als Hauptvertreter mit ihren zahlreichen verwandten Formen (10, p. 69), 2) das westatlantische (Holländisch-Westindien bis Florida und Cap Cod) mit C. xamachana, frondosa und van der Horsti. Dass $C$. xamachana von Jamaica und $C$. polypoides aus dem roten Meer einander sehr ähnlich sind, hat bereits MAAs betont. Ich habe oben nachgewiesen, dass die neue Species van der Horsti der ostafrikanischen $C$. andromeda von Djibuti sehr nahe steht.

Man wird vorläufig wohl noch immer gut tun, „sich vor weitgehenden Schlüssen zu hüten” (7, p. 46).

Angesichts des trostlosen Standes der Systematik des Genus Pelagia sowohl, als des Genus Cassiopeia, habe ich die Medusen von Curaçao nur mit Widerstreben als neue Species bezeichnet. Solange nicht eine auf umfangreichem von verschiedenen Fundorten herrührendem Material beruhende Revision sämmtlicher Arten der beiden Genera vorliegt, bleibt nichts anderes übrig, als jede einigermassen abweichende Form von neuem Fundort zu beschreiben. Selbst auf die Gefahr hin, dass diese "spec. nova” später einfach als "Lokalvarietät" angesehen wird. Möge daher auch solchen neuen Formen vielleicht nur ein ephemeres Dasein in der Litteratur beschieden sein, so haben doch derartige Beschreibungen und Neubenennungen den praktischen Vorteil, dass sie später in die Liste der Synonyma mit dem neuen Fundort aufgenommen werden müssen und der Gefahr entgehen, übersehen oder totgeschwiegen zu werden.

Im Falle Cassiopeia hat sich hier aufs Neue gezeigt, dass je 2 dieser Species von sehr entfernten Fundorten einander sehr nahestehen. Im Falle Pelagia wird eine Lücke in der geographischen Verbreitung ausgefüllt, so dass sich nunmehr ein zusammenhängendes räumlich abgegrenztes Verbreitungsgebiet ergibt. Dem Ziele, „eine möglichst geschlossene Reihe der vermeintlichen guten Arten” zu erhalten, ist man damit vielleicht doch um einen Schritt näher gekommen.

Im Anschlusse an die obige Beschreibung des Gefässystems bei Cassiopeia van der Horsti will ich einige Beobachtungen über Anomalien in der Ausbildung des Gefässystems bei Cassiopeia mitteilen, da mir augenblicklich Material verschiedener Species vorliegt. Die Anomalien betreffen die Ausbildung der Radiärcanäle und eines Ringcanals.

Das normale Verhalten scheint wohl zu sein, dass die Rhopalar- und Interrhopalarcanäle unverdickt, gleichmässig breit in ihrem ganzen Verlaufe vom Magen bis zur Peripherie ziehen, wobei die letzteren meist schwächer ausgebildet, kürzer sind und ein engeres Lumen haben. (Vergl. z.B. 7, Taf. IV, Fig. 30; 3, Taf. XXI; 1, Taf. XII, Fig. 2 und 5). In seltenen Fällen gabeln sich die Rhopalarcanäle, entweder unmittelbar an ihrer Ursprungsstelle am Magenrande oder mehr entfernt davon, oder 2 benachbarte Radialcanäle münden in einander und gabeln sich dann wieder (10, Taf. I, Fig. 1). Bei $C$. andromeda var. acycloblia nehmen die Radiärcanäle vom Centrum nach der Peripherie fortschreitend etwas an Breite zu; die Rhopalarcanäle sind am breitesten in unmittelbarer Nähe des Rhopaliums, die Interrhopalarcanäle lösen sich in der Nähe des Schirmrandes in ein mehroder minder engmaschiges Anastomosennetz auf.

Bei C. van der Horsti sind die Rhopalarcanäle zumeist auf den ersten Blick von den Interrhopalarcanälen durch die längliche sinusartige Verbreiterung im äusseren Drittel ihres Verlaufs zu unterscheiden (Textfig. 4). Die Sinus zeigen Spindelform und sind ganz unregelmässig abgegrenzt; bei 
einigen Exemplaren waren sie viel kürzer, mehr rhombisch oder rundlich. Aehnliches Verhalten zeigten die Rhopalarcanäle bei einigen Individuen von $C$. $a$. var. malayensis, wo die spindelförmige Verdickung mehr regelmässige Form zeigt und sich etwa im halben Abstand von der Peripherie findet. (Textfig. 5).

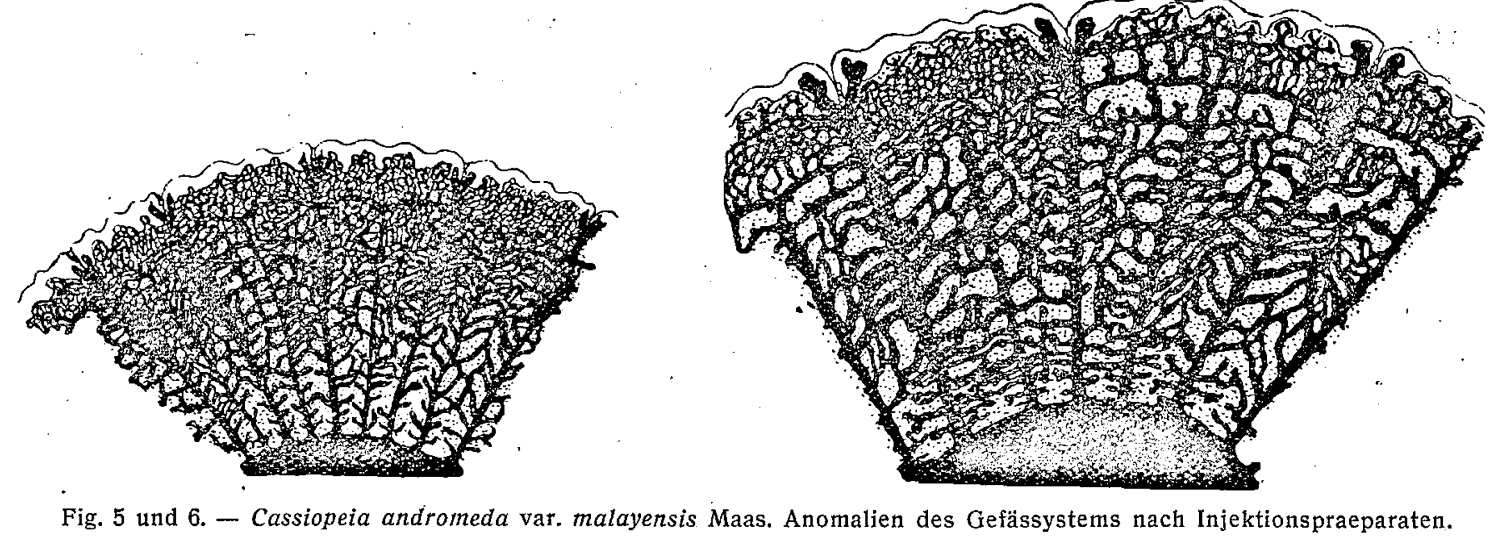

Nirgends ist ein ausgesprochener Ringcanal festzustellen. Wohl aber treten öfters, in benachbarten Sektoren, kleine nach aussen convex vorspringende Bogenstückchen im Anastomosennetz auf, guirlandenartig die Radiärcanäle mit einander verbindend, gerade dort, wo man etwa einen Ringcanal erwarten würde wie z.B. bei $C$. $a$. var. malayensis (Textfig. 5) oder mehr randständig (10, Taf. I, Fig. 1). Doch erfährt der Zusammenhang dieser rundlichen Bogenstückchen meist plötzlich in einem oder mehreren Sektoren eine Unterbrechung. - Eine andere Anomalie von $C$. a. var. malayensis bringt die Darstellung HAECKELS von einem doppelten Ringcanal bei $C$. ornata in Erinnerung $(6$, p. 571 u. 11, p. 42/43). Betrachten wir Textfig. 6. Die Rhopalarcanäle verdicken sich centrifugal, erreichen ihre grösste Breite in der Nähe der Rhopalien. Die beiden rechtsgelegenen Sektoren zeigen äussere periphere und mehr innerhalb gelegene nach aussen vorgewölbte Bogenstückchen von grösserer Breite als die übrigen Anastomosen, so dass man hier allenfalls an einen äusseren randständigen und inneren Ringcanal denken könnte. Im benachbarten Sektor (der 2. von links) ist jedoch keine Spur mehr davon zu sehen, das Anastomosennetz reicht, centrifugal stets feinmaschiger werdend, bis fast in die Randläppchen, während im 1. Sektor von links wieder eine Art innerer Ringcanal zu beobachten ist.

Ein Exemplar von C. van der Horsti weist an einer verheilten Wundstelle eine bemerkenswerte

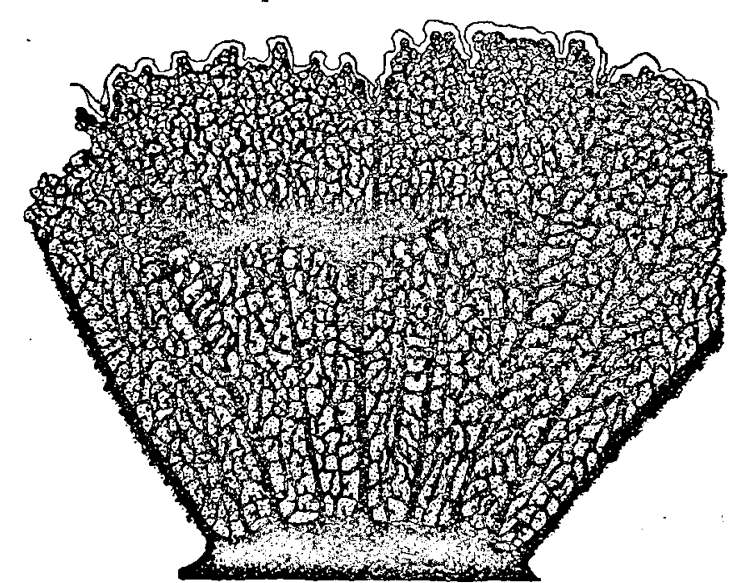

Fig. 7. - Cassiopeia van der Horsti nov. spec. Anomalie des Gefässystems des Schirmes an verheilter Wundstelle nach einem Injectionspraeparat. Anomalie auf (Textfig. 7). Ein Rhopalarcanal zeigt etwa in der Mitte seines nicht ganz geraden Verlaufes eine grosse T-förmige sinusartige Verbreiterung - eine Art Bruchstück eines Ringcanales - die sich über 2 Sektoren erstreckt und 3 Rhopalarcanäle mit einander verbindet.

Bei C. ornata Haeckel finden wir circa im äusseren Drittel des Verlaufes der Radiärcanäle „eine deutlich hervortretende Zone breiterer dickwandigerer Netzmaschen, die sowohl centripetal als centrifugal in ein feinmaschigeres Netz übergeht (11, p. 42/43).

Im ganzen genommen hat man wohl den Eindruck, dass bei Cassiopeia die Tendenz zur Ausbildung eines (secundären) Ringcanales vorhanden ist $(10$, p. $33-34)$.

Ueber die Entwicklung des Gefässystems von Cassiopeia ist nichts Näheres bekannt. HaRTLAuB erwähnt (5, p. 470) bei einem Exemplar von C. andromeda von $22 \mathrm{~mm}$ Schirmbreite, dass die Radiärcanäle noch unverzweigt und nur in ihren proximalen 2 Dritteln deutlich zu erkennen sind. Bei den jüngsten Exemplaren von $C$. acycloblia Schultze von 10-12 mm Schirmbreite sind die Radiärcanäle sämmtlich von gleicher Dicke, unverzweigt und nur in der inneren Hälfte ihres Verlaufes zu sehen; in der randständigen Zone sind sie noch nicht ausgebildet. Bei den jüngsten Stadien von 
C. a. var. malayensis Maas von circa $20 \mathrm{~mm}$ Schirmbreite sind die Radiärcanäle gleichfalls nur in den proximalen 2 Dritteln ihres Verlaufs zu erkennen. Etwas ältere Stadien zeigten bereits sämmtliche Canäle in ihrem ganzen Verlaufe ausgebildet, nur fiel es mir auf, dass die Verästelungen und Anastomosen in der äusseren Hälfte früher auftreten als in den centralen Teilen, wo die Radiärcanäle oft noch ganz unverästelt sind. Jüngere Exemplare zeigen im allgemeinen stets ein viel lockereres weitmaschigeres Anastomosennetz als ältere. Soviel steht also doch wohl fest, dass innerhalb der Entodermlamelle die Bildung der Radiärcanäle centrifugal vom Magen aus, die Bildung des Anastomosennetzes centripetal von dem Schirmrand aus erfolgt. 


\section{LITTERATUR-VERZEICHNIS.}

1) 1860. Agassiz, Louis. Contributions to the Natural History of the United States. Vol. III. Boston.

2) 1913. Bigelow, HenRY B. Medusae and Siphonophorae collected by the U. S. Fish-steamer "Albatross" in the North western Pacific. 1906. Proc. U. S. Nat. Mus. Vol. 14. Washington:

3) 1838. BRANDT, J. FR. Ausführliche Beschreibung der von C. H. Mertens auf seine Weltumseglung beobachteten Schirmquallen. Mem. Ac. Sc. St. Petersbourg. 6. ser. T. 4. sc. nat. Vol. II. Petersbourg.

4) 1905. Browne, E. T. Scyphomedusae. Fauna and Geography of the Maldive and Laccadive Archipelagoes. Vol. II, part 3. Cambridge.

5) 1909. Hartlaub, Cl. Ueber einige von Ch. Gravier in Djibuti gesammelte Medusen. Zool. Jahrb. Abt. Syst., Geogr. u. Biologie d. T. Bd 27. Jena.

6) 1879. HAEckel, E. Das System der Medusen. Mit Atlas. Jena.

7) 1903. MAAs, O. Die Scyphomedusen der Siboga-Expedition. Siboga-Expeditie, 11. Monogr. Leiden.

8) 1910. MAYER, A. G. Medusae of the world. III. The Scyphomedusae. Carnegie Inst. Washington.

9) 1898. Schultze, L. S. Rhizostomeen von Ambon. Denkschr. Jena. Ges. Naturw. Vol. 8. (Semon, Zool. Forsch. Vol. 5.) Jena. -

10) 1921. Stiasny, G. Studien über Rhizostomeen mit bes. Berücksichtigung der Fauna des. malay. Archipels u.s.w. Capita Zoologica, Deel I. 's Gravenhage.

11) 1922. - Ergebnisse der Nachuntersuchung einiger Rhizostomeen-Typen Haeckel's und Chun's aus dem Zoologischen Museum in Hamburg. Zoolog. Mededeel. Deel VII. Afl. 1. Leiden.

12) 1886. VANHÖFfEN, E. Untersuchungen über semaeostome und rhizostome Medusen. Bibl. Zool. Bd 1. Heft 3 . Cassel.

13) 1903. - Die acraspeden Medusen der Deutschen Tiefsee-Expedition. 1898-99. Ergeb. Deutsche Tiefsee-Exp. Jena.

14) 1829. Eschscholtz, Fr. System der Acalephen. Berlin.

15) 1923. HORST, C. J. VAN DER. Narrative of the voyage and short description of localities. In diesen Bijdragen.

Leiden, November 1922. 